\title{
A New Expression Model of Classical Electromagnetic Theory at the Micro Level
}

Jack Fan ( $\square$ jack.fan1993@gmail.com )

Institute of Theoretical Physics

Research Article

Keywords: charge, current, electric field, magnetic field, Maxwell.

Posted Date: November 24th, 2020

DOI: https://doi.org/10.21203/rs.3.rs-112346/v1

License: (c) (i) This work is licensed under a Creative Commons Attribution 4.0 International License. Read Full License 


\title{
A New Expression Model of Classical Electromagnetic
}

\author{
Theory at the Micro Level
}

\author{
Jack Fan \\ Institute of Theoretical Physics, Yunnan, Kunming, 650000 P. R. China
}

\begin{abstract}
:
Because Maxwell's classical electromagnetic theory is a macro-electromagnetic theory based on the cognition of the 19th century and before the 19th century, and because the physics of the 20th century has gained new cognition in the microscopic field, considering that it is now the 21 st century. Therefore, this article attempts to establish a set of micro-expression theory of Maxwell's macro-electromagnetism theory based on the new cognitions obtained at the micro-level since the 20th century, in order to promote the further development of Maxwell's classical electromagnetism theory in accordance with the cognition of the times.
\end{abstract}

Keywords: charge; current; electric field; magnetic field; Maxwell.

\section{1: Introduction}

What is the origin of charge on the micro level? What is the origin of electric current at the micro level? What is the origin of voltage at the micro level? What is the origin of the electric field at the micro level? What is the origin of the magnetic field at the micro level? What is the origin of electromagnetic force at the micro level? What is the origin of electromagnetic waves at the micro level? What is the origin of magnetic monopole on the microscopic level? This has always been the mystery of physics. At the same time, looking at the relevant literature, we can understand that predecessors have made a lot of efforts in these fields [1-13]. Therefore, I also want to contribute in my own way.

Maxwell's classical electromagnetic theory is a set of macro-electromagnetic theory based on the cognition of the 19th century. It can describe various macro-electromagnetic phenomena well and has made great achievements, but it has always lacked a micro-expression theory. Based on the new understanding of quantum mechanics and atomic physics in the 20th century at the micro level, I tried to establish a new theory of the micro expression of Maxwell's macro-electromagnetic theory, to make up for the lack of Maxwell's macro-electromagnetism theory at the micro level, and tried to use it to explain some unsolved problems of macro-electromagnetic theory.

Among them, I gain a new understanding of current and voltage by interpreting the origin of electric charge on a microscopic level. At the same time, I also interpret the origins of electric and magnetic fields from a microscopic level to obtain a feasible way of using magnetic field properties to explain electric field properties.

\section{2: New definition of charge, current, voltage and resistance at the micro level}

According to the classical electromagnetic theory [14], the conduction current is the quantity of charges that flow across a section of a conductor per unit time, which can be expressed mathematically as follows:

$$
I=\frac{d q}{d t}
$$

Since the conduction current is based on a macroscopic model theory proposed by the Drude-Lorentz model and Drude-Sommerfeld model [15] (It uses the transfer of charge as a macroscopic physical quantity to explain the current). Consider that we don't know what the origin of the charge is on the microscopic level. 
Therefore, I tried to interpret the origin of electric charge at the micro level to gain a new understanding of the current at the micro level, so as to establish a set of microscopic expression models of the conduction current theory.

Based on the new understanding of atomic physics [16] and quantum mechanics [17] at the micro level, especially according to the photoelectric effect, the energy level transition of electrons can generate electric current. In other words, electrons generate electric current by transferring energy specific to the energy level difference. Considering that the energy of the energy level difference of electrons is a manifestation of electron momentum, that is to say, at the microscopic level, the essence of electron transfer charge to generate current is the electron transfer momentum to generate momentum flow. In other words, charge is a manifestation of electron momentum, and current is a momentum flow. That is to say, the current is the transfer of momentum $P$ transfer between electrons. Because electrons movement around the nucleus, so here the electronic momentum $P$ is the electronic angular momentum increment or impulse $u$, namely $P \Rightarrow u$. The current $I$ can be expressed as the quantity of electronic angular momentum increment or impulse $u$ that flow across a section of a conductor per unit time. Thus, the origin of the charge is the increase or impulse of the electron angular momentum $u$. Then, conduction current can become conduction Momentum flow $I \Rightarrow I_{p}$ :

$$
I \Rightarrow I_{p}=\frac{d p}{d t}=\frac{d u}{d t}
$$

According to (1) and (2), it can be seen that at the macro level, the Drude-Lorentz model and Drude-Sommerfeld model use the transfer of charge to explain the current; at the micro level, we should use the transfer of momentum to explain the current. Therefore, at the micro level, we have given a new definition of current. Of course, we can see from the above formula that when the current has no momentum loss during the transmission process, the transmission process will become an ideal superconductor. Therefore, the above new definition of current can also provide a theoretical explanation for superconductivity at the micro level. This explanation seems to be more concise than the BCS theory.
Then, the momentum flow density can be expressed as:

$$
J_{p}=\frac{d p}{d t \cdot d s_{\perp}}
$$

If the number of electrons per unit volume in the conductor is $n$, then $\rho=p n \quad(p$ is electron momentum). Then the momentum flow density in the conductor is:

$$
J_{p}=n p v
$$

The relationship between the momentum flow density and momentum flow in the surface $s$ is:

$$
I_{p}=\iint_{s} j_{p} \cdot d s
$$

On closed surfaces, it is:

$$
I_{p}=\oiint_{s} j_{p} \cdot d s
$$

The continuity equation of its momentum flow is:

$$
I_{p}=\oiint_{s} j_{p} \cdot d s=-\frac{d p_{\mathrm{int}}}{d t}
$$

Because the current is the momentum flow, so, the electromotive force is the impulse force of the electron, expressed as:

$$
\varepsilon=\int \frac{d p}{d t} d t
$$

Because the current is the momentum current produced by the impulse of the electron angular momentum. Therefore, the voltage is the orbital potential difference or energy level difference or potential pressure:

$$
U_{a b}=E_{a}-E_{b}=\varepsilon
$$

Therefore, I gave a new definition of voltage at the micro level, and prove that the origin of voltage is a potential pressure (potential difference or energy level difference).

Considering that current is momentum flow, resistance can be regarded as a momentum resistance $R \Rightarrow R_{p}$.

Summary: Through the above derivation, we can see that based on the new understanding of atomic physics and quantum mechanics at the microscopic level in the 20th century. At the micro level, we 
should understand current as momentum flow, and electrons generate current by transferring momentum; charge should be understood as a manifestation of electronic momentum, specifically in the form of electronic momentum or the increment (impulse) of electronic angular momentum; voltage should be understood as the potential difference (energy level difference) of electrons. Thus, we have proposed a new understanding of current, charge and voltage at the micro level. In addition, according to the above derivation, it can be seen that the conducted momentum flow theory proposed at the micro level can explain the related phenomena that the conducted current theory proposed at the macro level can explain. It can also explain some phenomena that the conduction current cannot explain (for example, the origin of charges, currents, and voltages at the micro level). Therefore, this means that the conducted momentum flow theory proposed at the micro level has more extensive compatibility than the conducted current theory proposed at the macro level. Therefore, this represents an advancement in physics.

\section{Discussion and explanation:}

The conduction current theory proposed on the macro level has two defects that can be solved (compatible) by the conduction momentum flow theory proposed on the micro level. One is that according to the photoelectric effect, electrons absorb the energy of photons to generate current through energy level transitions, because the energy level difference between photons and electrons does not have charge properties, but only has momentum properties. This cannot be explained by the theory of conduction current, which will lead to the generation of charges for no reason, can only be explained by the theory of conduction momentum flow. The other is based on the heating effect of power transmission loss (such as the heating phenomenon of mobile phones and computers). The current lost during the transmission process becomes heat energy, and this thermal energy has no charge attribute. If the conduction current theory is used to explain the heating phenomenon of transmission loss, which will cause the charge to disappear for no reason. This can only be explained by the momentum flow theory of conduction loss.

The above two cases can fully illustrate the limitations of the theoretical interpretation of the conducted current theory proposed at the macro level. We need a set of more widely compatible theories.

\section{3: The origin and new definition of Electric field, magnetic field, electromagnetic force and electromagnetic wave at the micro level}

Based on the new cognition of atomic physics, we know the atomic model, know the existence of electrons, know the electron spin, know the movement of electrons around the nucleus, and know the existence of electron magnetic moments (Note: This is a new cognition born in the 20th century. There was no such cognition in Maxwell's time). According to the classical electromagnetic theory, the electric field intensity is expressed as:

$$
E=\frac{F}{q}
$$

As is shown above, the nature of the charge is the impulse $u$ of the electronic angular momentum. According to classical electromagnetic theory and quantum optics theory [18], the electric field is generated by the charge, and the photon is the medium particle of the electric field. It follows that the electric field force is the force produced by the collision of electronic angular momentum impulses with photons. This force is an impulse moment, expressed as $M=r \times u$. The impulse of the electronic angular momentum is generated by electron spin and orbital motion, so, the field source of the electric field is the electron magnetic moment, and the field source particles are electrons $e$, namely $q \Rightarrow e$. Given that the magnetic field is generated by the molecular magnetic moment $\sum m \neq 0$, while the magnetic moment is generated by the electron spin angular momentum and orbital angular momentum, and also given that $t$ the photon is the medium particles of the magnetic field, then the magnetic field force can also be seen as the force produced by the collision of electronic angular momentum impulses with photons. This force is also an impulse moment, expressed as $M=r \times u$. Accordingly, the field source of the magnetic field is also the electron magnetic moment, and the field source particles are also electrons $e$. Therefore, we can deduce that the electric field $E$ 
and the magnetic field $H$ have the same field source, which is the electron $e$. So, we can get a powerful equation that explains that a magnetic field and an electric field have the same field source:

$$
\left\{\begin{array}{l}
H=\frac{F}{e} \\
E=\frac{F}{e}
\end{array}\right.
$$

According to the above derivation, since the electric field and the magnetic field have the same field source, the electric field and the magnetic field belong to the same object. This signifies that the nature of the electric field is the magnetic field, so the nature of the electric field force is the magnetic force and the acting force of the positive and negative charges is the acting force of the two magnetic poles. Therefore, we explain the electric field from the aspect of the magnetic field, and explain the acting force of positive and negative charges from the aspect of the magnetic force. Electromagnetic waves can be interpreted as media fluctuations generated by electronic collision media. The amount of charge measured by Millikan's 'oil-drop' experiment can be interpreted as the amount of action produced by the electron magnetic moment. The positive and negative charges can be interpreted as the behavior of the two magnetic poles of the magnetic field. (Note: This is a new perspective on electromagnetics based on the new cognition of atomic physics obtained at the micro level in the 20th century. Because the existence of magnetic moment has not been discovered in Maxwell's time, the cognitive level based on Maxwell's time is not enough to support the use of magnetic moment to explain the origin of electric and magnetic fields)

Since we have explained the electric field from the aspect of the magnetic field, we need to explain the nature of magnetic and magnetic forces. For the nature of the magnetic force, we can assume from the kinetic point of view that the impulse of the angular momentum impinging on the photon of the medium causes the photon vortex to move, which in turn produces a medium impulse moment (similar to fan blowing). The medium impulse moment is the magnetic force, and the essence of the magnetic force line can be regarded as the cyclotron motion path of the medium photon. Thus, the magnetic force represents the cross product of the impulse $u$ applied by the electron to the photon of the medium with the vector of the photon displacement vector $r$, which can be expressed mathematically as:

$$
M=r \times u
$$

Magnetic field can be defined as "a vortex force system generated by photon cyclotron motion of electrons colliding with multiple spins", that is the superposition state of the magnetic moment. Its mathematical expression is:

$$
M=\sum m
$$

If the magnetic field line is used to express the magnetic field, then the magnetic field can also be expressed with the geometric changes of field strength and magnetic flux:

$$
\Phi_{H}=\iint_{s} H \cdot d s
$$

In this way, we defined the nature of magnetic field and magnetic force, and thus unraveled the mystery about the intrinsic nature of electromagnetic field and electromagnetic force.

According to the law of Ampere Circle $\oint_{L} B \cdot d l=\mu_{0} \sum_{i=1}^{n} I_{i}$, the relationship between the magnetic field strength and the current in the current-carrying conductor can be expressed as:

$$
\oint_{L} H \cdot d l=\mu_{0} \sum_{i=1}^{n} I_{i}
$$

Taking into account that the field source of the magnetic field is the electron $e$, the force applied on static electrons in the magnetic field is:

$$
F=e H
$$

The force applied on moving electrons in the magnetic field is:

$$
F=e v \times H
$$

The total force acting on the current-carrying conductor in the magnetic field is:

$$
F=\int_{L} d F=\int_{L} I d l \times H
$$

Taking into account that the field source of the magnetic field is the electron $e$, and the impulse momentum produced by per unit electron is $M_{e}$. With reference to Biot-Savart law, it indicates that the physical meaning of current element is the electronic impulse moment $I d l \Rightarrow M_{e}$. Then the 
magnetic field strength generated by the electron at any point in the space can be expressed as:

$$
d H=k \frac{d M_{e} \times e_{r}}{r^{2}}=k \frac{I d l \times e_{r}}{r^{2}}
$$

The vector integral form is:

$$
H=\int_{L} d H=k \int_{L} \frac{d M_{e} \times e_{r}}{r^{2}}
$$

The magnetic interaction between two electrons can be expressed as:

$$
d F_{1 \rightarrow 2}=k \frac{d M_{e 2} \times\left(d M_{e 1} \times e_{r}\right)}{r^{2}}
$$

When the vector is not considered, the acting forces between two electrons can be idealized as $F_{1 \rightarrow 2}=\frac{1}{4 \pi \varepsilon_{0}} \frac{e_{2} e_{1} r}{r^{3}}$. The equivalent Coulomb's law thus can be derived:

$$
F_{1 \rightarrow 2}=\frac{1}{4 \pi \varepsilon_{0}} \frac{e_{2} e_{1} r}{r^{3}} \Leftrightarrow \frac{1}{4 \pi \varepsilon_{0}} \frac{q_{2} q_{1} r}{r^{3}}
$$

It can be seen from the above that we can deduce the equivalent Coulomb's law from the angle of the magnetic field, and prove that the Coulomb electrostatic field is a current element magnetic field. Thus, we have proposed a feasible way to explain the electrostatic field with a magnetic field.

\section{Thus, we can come up with a prediction:}

"The frictional electrification phenomenon is the friction generating current (momentum flow) and the magnetic field".

\section{The mechanism is:}

Friction causes the electron to direct the collision motion to generate a momentum flow (current), and the electron momentum flow (current) polarizes the electron magnetic moment to generate a magnetic field (this is an electromagnet).

The rubbed object has the property of attracting light and small objects. The essence is that the friction generates a current, and the current acts on the electron magnetic moment of the small and small object, causing the electron magnetic moment to be polarized to display magnetic properties and generate a magnetic field. The attraction phenomenon is the interaction between two magnetic fields. Therefore, the frictional electrification phenomenon is the friction generating current and magnetic field.

\section{Therefore, we can draw 3 predictions:}

A: The rubbed ruler has a magnetic field that can cause small magnetic needle deflection.

$\mathrm{B}$ : The rubbed ruler has electromagnetic radiation.

C: The electromagnet can attract light and small objects (paper scraps).

\section{Experimental results:}

A: In the experiment, the rubbed ruler can trigger the deflection of the small magnetic needle (welcome to repeat this experiment), and the strength of the magnetic field generated by the rubbed ruler is $0.2 G s$.

B: The rubbed ruler has an electromagnetic radiation intensity of $0-3160 \mathrm{w} / \mathrm{cm}^{2}$.

\section{Experimental video links (A and B):}

\section{https://www.ixigua.com/i6555401121496564231/}

Note: I uploaded the experiment video to the web page for review needs. It can be deleted after the review is passed.

C: When the electromagnetic radiation value is $0-8181 \mathrm{w} / \mathrm{cm}^{2}$, the electromagnet can produce more intense and significant experimental phenomena of attracting shredded paper.

\section{Experimental verification method $(\mathbf{C})$ :}

We can confirm by observing whether there is an experimental phenomenon in the strong magnetic field that can attract small and light objects (crushed paper).

Discussion: If we see the phenomenon that a strong magnetic field can attract light and small paper scraps, the theory is correct and we will need to revise the textbook. If we do not see such a phenomenon, the theory is wrong. (Although we have carried out experiments to verify our conjecture, we still invite you to verify our theory through repeated experiments).

Experimental equipment: power supply(AC), voltage regulator, after the power to generate magnetic field copper coil, paper, field strength measuring instrument

Experimental steps: 
1: Series the power supply(AC), voltage regulator and copper coil

2: Sprinkle paper scraps around the copper coil

3: Turn on the power supply, through the voltage regulator to control the current, observe the copper coil around the magnetic field can attract paper scraps.

4: In the experiment, we can see that the greater the current, the greater the intensity of the magnetic field, attracting the more intense. the intensity of electromagnetic radiation (value range is $0-8181 \mathrm{w} /$ $\mathrm{cm}^{2}$ ).

This experiment proves that electromagnets (strong magnetic fields) can also attract shredded paper (or electrostatic attraction).

\section{Experimental video link:}

https://www.ixigua.com/i6555403150042333710/

Note: I uploaded the experiment video to the web page for review needs. It can be deleted after the review is passed.

\section{Discussion:}

According to the conclusions herein, even without charge, the magnetic field can attract shredded paper. However, because ordinary magnets don't attract shredded paper and other light and small objects, I need to clarify. Here, the reason why ordinary magnets cannot attract shredded paper is that the magnetic force is small and the magnetization ability is weak. The rubbed ruler can attract shredded paper because friction generates current (momentum flow), current can re-arrange the electron magnetic moment of small and light objects and generate magnetic field, so the rubbed ruler is more likely to magnetize and attract paper scraps. (Note: Because the current can cause the electrons of light and small objects to be rearranged, and the electrons have magnetic moments, when the electrons are rearranged, the magnetic moment of the electrons will be rearranged again. Therefore, the magnetization ability of the current is stronger than that of ordinary permanent magnets. Can magnetize and attract shredded paper.).

In fact, because any object has an electronic magnetic moment, any object has an internal magnetic property. Therefore, in theory, as long as the magnetic field strength is strong enough, the magnet can attract any object. Therefore, we can use a powerful magnet to achieve the phenomenon of attracting shredded paper. In fact, powerful magnets can also attract hanging apples, tomatoes and kiwis, as well as attracting burnt matches (I can provide experimental videos).

\section{Experimental video link:}

https://www.ixigua.com/i6555404509365928455/

Note: I uploaded the experiment video to the web page for review needs. It can be deleted after the review is passed.

The force exerted by the current carry conductor $L_{1}$ on the electron $e_{2}$ is:

$$
d F_{1 \rightarrow 2}=M_{e 2} \times \int_{L_{1}} \frac{d M_{e 1} \times e_{r}}{r^{2}}
$$

The force exerted by the current carrying conductor $L_{1}$ on the current carrying conductor $L_{2}$ is:

$$
F_{1 \rightarrow 2}=\int_{L_{2}} d F_{1 \rightarrow 2}=\int_{L_{2}} \int_{L_{1}} \frac{d M_{e 2} \times\left(d M_{e 1} \times e_{r}\right)}{r^{2}}
$$

On the contrary:

$$
F_{L_{2} \rightarrow L_{1}}=-F_{L_{1} \rightarrow L_{2}}
$$

From the above, we can extrapolate that the magnetic field excited by the impulse of the electronic angular momentum follows the inverse square of the distance.

The moment of forces in the magnetic field can be expressed as:

$$
M=m \times H
$$

According to Faraday's law of electromagnetic induction, we can work out the relationship equation between EMF and magnetic field strength:

$$
\varepsilon_{i}=-\frac{d \Phi}{d t}=-\iint_{s} \frac{\partial H}{\partial t} \cdot d s
$$

It can be inferred from the above deduction that we can theoretically explain all the electric field phenomena from the aspect of the magnetic field, and predict that triboelectrification is a result of friction generating a magnetic field, which is another major breakthrough point in this paper.

Discussion: If triboelectrification is essentially a phenomenon where friction produces a magnetic field, then the unipolar electrostatic field is actually a dipole magnetic field. We can verify this by seeing if the charged scraps in the 
triboelectrification phenomenon have the magnetic properties of the two poles.

\section{4: A new expression of Maxwell's equations at the microscopic level}

According to the above deduction, the origin (essence) of the electric field at the microscopic level should be re-understood as the electric current element magnetic field. In this way, the same object cannot be transformed into each other, so we need to give a new expression of Maxwell's equations at the micro level.

According to the above-mentioned derivation of Faraday's law of electromagnetic induction, the electromotive force in the magnetic field is:

$$
\varepsilon_{i}=-\frac{d \Phi}{d t}=-\iint_{s} \frac{\partial H}{\partial t} \cdot d s
$$

The electromotive force $\varepsilon_{i}$ here represents the impulse force of the electron or the energy level difference of the electron (potential difference).

The fourth equation of Maxwell's equations is:

$$
\oint_{L} H \cdot d l=I_{o \text { int }}+\iint_{s} \frac{\partial D}{\partial t} \cdot d s
$$

Considering that the current density of electric displacement is $J_{d}=\frac{\partial D}{\partial t}$, and the current density of the conduction momentum flow is $J_{p}=\frac{d p}{d t \cdot d s_{\perp}}$, then the relationship between the electric displacement $D$ and electron momentum $p$ can be expressed as $\partial D=\partial p$, so the above equation can be improved as follows:

$$
\oint_{L} H \cdot d l=I_{o \text { int }}+\iint_{s} \frac{\partial p}{\partial t} \cdot d s
$$

This shows that changing electron momentum can generate a magnetic field.

Consider that the electric field is a manifestation of the magnetic field of the current element, and considering that the electric field and the magnetic field have the same field source, namely the electron $e$, the equations of the Maxwell's equations of the electric field and the magnetic field can be overlooked. Therefore, based on the new understanding at the micro level, the micro expression of Maxwell's equations is:

$$
\left\{\begin{array}{l}
\varepsilon_{i}=-\iint_{s} \frac{\partial H}{\partial t} \cdot d s \\
\oint_{L} H \cdot d l=I_{o \text { int }}+\iint_{s} \frac{\partial p}{\partial t} \cdot d s
\end{array}\right.
$$

It can be seen from the above derivation that a changing magnetic field can generate an electromotive force, (electron impulse force or electron energy level difference or potential pressure), and a changing electron momentum can generate a magnetic field. Thus, by introducing new understandings of atomic physics and quantum mechanics at the micro level, I proposed a feasible theory of using magnetic fields to explain the properties of electric fields. This fills a gap in quantum electrodynamics.

\section{5: Conclusion}

This article aims to establish a new theory of Maxwell's macro-electromagnetism theory micro-expressions to make up for some of the deficiencies of the macro-electromagnetism theory at the micro level, and try to use it to solve some problems that have not been solved by macro-electromagnetism theory to promote the further development of electromagnetism. Finally, based on the microscopic expression of Maxwell's electromagnetic theory, we propose a feasible interpretation of the origins of charge, current, voltage, electric field and magnetic field. This fills a gap in QED from a certain angle.

In addition, since Maxwell unified electromagnetics, we all firmly believe that electric and magnetic fields are different manifestations of the same object, but there has been a lack of a final proof. Today I was fortunate to find that the electric field is a form of current element magnetic field. We can use the magnetic field to explain all the properties of the electric field, thus proving our long-term conjecture.

For Maxwell's era, Maxwell's focus was on unifying the electric and magnetic fields on a macro level and establishing their mutual transformation relationship. For our time, our focus is to further use one of the attributes to explain or be compatible 
with the other at the micro level, so as to finally prove that they are the same object and promote the further development of Maxwell's classical electromagnetic theory.

\section{Acknowledgement}

First of all, I thank my good brother Hao-yang Zhang from the Department of Physics of Oxford University for the discussion.

Secondly, I would like to thank the Nobel Prize winner in 1973, Professor Josephson of the Cavendish Laboratory of the University of Cambridge, for the one-month discussion of my paper and his valuable suggestions for my experiment.

\section{References}

[1] Eringen A C, Maugin G A. Microscopic Electromagnetic Theory[M]// Electrodynamics of Continua I. Springer New York, 1990.

[2] Shadid W G T. Two New Theories for the Current Charge Relativity and the Electric Origin of the Magnetic Force Between Two Filamentary Current Elements[J]. IEEE Access, 2016, 4:4509-4533.

[3] Ivezic T. Nature of Electric and Magnetic Fields; How the Fields Transform[J]. Physics, 2015.

[4] Ioffe A Z D A F. Origin of the Magnetic Field[C]// International Astronomical Union Colloquium. Cambridge University Press, 1986.

[5] Ferris M R, Gratus J. The origin of the Schott term in the electromagnetic self-force of a classical point charge $[\mathrm{J}]$. Journal of Mathematical Physics, 2011, 52(9):092902.

[6] Qi X L, Li R, Zang J, et al. Inducing a Magnetic Monopole with Topological Surface States[J]. Science, 323.

[7] Niemi A J, Paranjape M B, Semenoff G W. Electric Charge of the Magnetic Monopole[J]. Phys.rev.lett, 1984, 53(53):515-518.

[8] Bhattacharya R. Comment on the CP-violating electric charge of the magnetic monopole[J]. Zeitschrift Für Physik C Particles \& Fields, 1986, 32(2):215-220.

[9] Kazama Y, Yang C N, Goldhaber A S. Scattering of a Dirac particle with charge $\mathrm{Z}$ e by a fixed magnetic monopole[J]. Physical Review D, 1976, 15:8(15):2287-2299.

[10] Goldhaber A S, Singh H, Parwani R. On the fractional electric charge of a magnetic monopole at nonzero temperature $[\mathrm{J}]$. Physics Letters B, 1996, 386(1-4):207-210.

[11] Jaubert L D C, Holdsworth P C W. Magnetic monopole dynamics in spin ice[J]. Journal of Physics: Condensed Matter, 2011, 23(16): 164222.

[12] Sivers, Dennis. Possible Binding of a Magnetic Monopole to a Particle with Electric Charge and a Magnetic Dipole Moment[J]. Physical Review D, 1970, 2(9):2048-2054.

[13] Davidson A, Guendelman E I. Electric monopole with internal magnetic-monopole-like structure[J]. Physics Letters B, 1990, 251(2):250-253.

[14] $\sim$ Halliday D, $\sim$ Resnick R, Walker J. Fundamentals of Physics[J]. Physics Today, 2003, 25(4):53-54.

[15] Ashcroft N W, Mermin N D. SOLID STATE PHYSICS[M]. HOLT, RINEHART AND WINSTON, 1976.

[16] Datz S, Drake G W F, Gallagher T F, et al. Atomic physics[J]. Rev.mod.phys, 1999, 71(2):377-407.

[17] Elton, B L R. Quantum Mechanics (Non-Relativistic Theory) [J]. Physics Bulletin, 1958, 9(10):270-271.

[18] Scully M O, Zubairy M S, Milonni P W. Quantum Optics[J]. Physics Today, 1998, 51(10):90-92. 